

\title{
On twists of modules over noncommutative Iwasawa algebras
}

\author{
Somnath Jha, Tadashi Ochiai and Gergely Zábrádi
}

\begin{abstract}
It is well known that, for any finitely generated torsion module $M$ over the Iwasawa algebra $\mathbb{Z}_{p}[[\Gamma]]$, where $\Gamma$ is isomorphic to $\mathbb{Z}_{p}$, there exists a continuous $p$-adic character $\rho$ of $\Gamma$ such that, for every open subgroup $U$ of $\Gamma$, the group of $U$-coinvariants $M(\rho)_{U}$ is finite; here $M(\rho)$ denotes the twist of $M$ by $\rho$. This twisting lemma was already used to study various arithmetic properties of Selmer groups and Galois cohomologies over a cyclotomic tower by Greenberg and Perrin-Riou. We prove a noncommutative generalization of this twisting lemma, replacing torsion modules over $\mathbb{Z}_{p}\left[[\Gamma]\right.$ by certain torsion modules over $\mathbb{Z}_{p}[[G]$ with more general $p$-adic Lie group $G$. In a forthcoming article, this noncommutative twisting lemma will be used to prove the functional equation of Selmer groups of general $p$-adic representations over certain $p$-adic Lie extensions.
\end{abstract}

\section{Introduction}

Let us fix an odd prime $p$ throughout the paper. We denote by $\Gamma$ a $p$-Sylow subgroup of $\mathbb{Z}_{p}^{\times}$. For a compact $p$-adic Lie group $G$ and the ring $\mathcal{O}$ of integers of a finite extension of $\mathbb{Q}_{p}$, we denote the Iwasawa algebra $\mathcal{O} \llbracket G \rrbracket$ of $G$ with coefficient in $\mathcal{O}$ by $\Lambda_{\mathcal{O}}(G)$.

In this article, we study $\Lambda_{\mathcal{O}}(G)$-modules, motivated by [Coates et al. 2005]. More precisely, we study specializations of certain $\Lambda_{\mathcal{O}}(G)$-modules by two-sided ideals of $\Lambda_{\mathcal{O}}(G)$. Recall that the paper [Coates et al. 2005] establishes a reasonable setting of noncommutative Iwasawa theory in the following situation.

(G) $G$ is a compact $p$-adic Lie group which has a closed normal subgroup $H$ such that $G / H$ is isomorphic to $\Gamma$.

S. Jha gratefully acknowledges the support of a JSPS postdoctoral fellowship and a DST INSPIRE faculty award grant. T. Ochiai is partially supported for this work by KAKENHI (Grant-in-Aid for Exploratory Research: Grant Number 24654004, Grant-in-Aid for Scientific Research (B): Grant Number 26287005). G. Zábrádi was supported by a Hungarian OTKA Research grant K-100291 and by the János Bolyai Scholarship of the Hungarian Academy of Sciences.

MSC2010: primary 11R23; secondary 16S50.

Keywords: Selmer group, noncommutative Iwasawa theory. 
According to the philosophy of [Coates et al. 2005], for a reasonable ordinary $p$-adic representation $T$ of a number field $K$ and a pair of compact $p$-adic Lie groups $H \subset G$ satisfying the condition $(\mathrm{G})$, the Pontryagin dual $\mathcal{S}_{A}^{\vee}$ of the Selmer group $\mathcal{S}_{A}$ of the Galois representation $A=T \otimes \mathbb{Q}_{p} / \mathbb{Z}_{p}$ over a Galois extension $K_{\infty} / K$ with $\operatorname{Gal}\left(K_{\infty} / K\right) \cong G$ seems to be a nice object. The $\Lambda_{\mathcal{O}}(G)$-module $\mathcal{S}_{A}^{\vee}$ divided by the largest $p$-primary torsion subgroup $\mathcal{S}_{A}^{\vee}(p)$ is conjectured to belong to the category $\mathfrak{n}_{H}(G)$ which consists of finitely generated $\Lambda_{\mathcal{O}}(G)$-modules $M$ such that $M$ is also finitely generated over $\Lambda_{\mathcal{O}}(H)$. From such arithmetic background, we are led to study finitely generated $\Lambda_{\mathcal{O}}(G)$-modules for a compact Lie group $G$ with $H \subset G$ satisfying the condition $(\mathrm{G})$.

On the other hand, for any open subgroup $U$ of $G$ and for any arithmetic module $\mathcal{S}_{A}^{\vee}$ as above, the largest $U$-coinvariant quotient $\left(\mathcal{S}_{A}^{\vee}\right)_{U}$ is expected to be related to the Selmer group of $A$ over a finite extension $L$ of $K$ with $\operatorname{Gal}(L / K) \cong G / U$. As remarked above, we have the following fact (Tw) when $G=\Gamma$ (i.e., when $H=1$ ) which was used quite effectively in the work of Greenberg [1989] and Perrin-Riou [2003].

(Tw) For any finitely generated torsion $\Lambda_{\mathcal{O}}(\Gamma)$-module $M$, there exists a continuous character $\rho: \Gamma \rightarrow \mathbb{Z}_{p}^{\times}$such that the largest $U$-coinvariant quotient $\left(M \otimes_{\mathbb{Z}_{p}}\right.$ $\left.\mathbb{Z}_{p}(\rho)\right)_{U}$ of $M \otimes_{\mathbb{Z}_{p}} \mathbb{Z}_{p}(\rho)$ is finite for every open subgroup $U$ of $\Gamma$, where $\mathbb{Z}_{p}(\rho)$ is a free $\mathbb{Z}_{p}$-module of rank one on which $\Gamma$ acts through the character $\Gamma \stackrel{\rho}{\rightarrow} \mathbb{Z}_{p}^{\times}$.

We call such a statement (Tw) a twisting lemma. In this commutative situation of $G=\Gamma$, the twisting lemma is proved in a quite elementary way. For example, we consider the characteristic ideal $\operatorname{char}_{\mathcal{O} \| \Gamma \rrbracket} M$. If we take a $\rho$ such that the values $\rho(\gamma)^{-1} \zeta_{p^{n}}-1$ do not coincide with any roots of the distinguished polynomial associated to $\operatorname{char}_{\mathcal{O} \llbracket \Gamma \rrbracket} M$ when natural numbers $n$ and $p^{n}$-th roots of unity $\zeta_{p^{n}}$ vary, the twisting lemma is known to hold.

If we have a twisting lemma in a noncommutative setting, it seems quite useful for some arithmetic applications for noncommutative Iwasawa theory. On the other hand, for a noncommutative $G$, it was not clear what to do to prove the twisting lemma because we cannot talk about "roots of characteristic polynomials" as we did in commutative setting. We finally succeeded in proving the twisting lemma which is stated as our Main Theorem below.

For a $\Lambda_{\mathcal{O}}(G)$-module $M$ and a continuous character $\rho: \Gamma \rightarrow \mathbb{Z}_{p}^{\times}$, we denote by $M(\rho)$ the $\Lambda_{\mathcal{O}}(G)$-module $M \otimes_{\mathbb{Z}_{p}} \mathbb{Z}_{p}(\rho)$ with diagonal $G$-action.

Main Theorem. Let $G$ be a compact p-adic Lie group and let $H$ be a closed normal subgroup such that $G / H$ is isomorphic to $\Gamma$. Let $M$ be a $\Lambda_{\mathcal{O}}(G)$-module which is finitely generated over $\Lambda_{\mathcal{O}}(H)$. 
Then there exists a continuous character $\rho: \Gamma \rightarrow \mathbb{Z}_{p}^{\times}$such that the largest $U$ coinvariant quotient $M(\rho)_{U}$ of $M(\rho)$ is finite for every open normal subgroup $U$ of $G$.

We give some examples of a pair $H \subset G$ satisfying the condition $(\mathrm{G})$ and a $\Lambda_{\mathcal{O}}(G)$-module $M$ which should appear in arithmetic applications.

Examples. (1) Let us choose a prime $p \geq 5$. Let $E$ be a non-CM elliptic curve over $\mathbb{Q}$ with good ordinary reduction at $p$. Take $K=\mathbb{Q}(E[p])$ and set $K_{\infty}=$ $\mathbb{Q}\left(\bigcup_{n \geq 1} E\left[p^{n}\right]\right)$. Then by a well known result of Serre, $\operatorname{Gal}\left(K_{\infty} / K\right)$ is an open subgroup of $\mathrm{GL}_{2}\left(\mathbb{Z}_{p}\right)$. By Weil pairing, the cyclotomic $\mathbb{Z}_{p}$ extension $K_{\text {cyc }}$ of $K$ is contained in $K_{\infty}$. We denote $\operatorname{Gal}\left(K_{\infty} / K\right), \operatorname{Gal}\left(K_{\infty} / K_{\text {cyc }}\right)$ and $\operatorname{Gal}\left(K_{\text {cyc }} / K\right)$ by $G, H$ and $\Gamma$ respectively. The pair $H \subset G$ satisfies the condition (G).

Let us consider the Pontryagin dual $\mathcal{S}_{A}^{\vee}$ of the Selmer group $\mathcal{S}_{A}$ of the Galois representation $A=T_{p} E \otimes \mathbb{Q}_{p} / \mathbb{Z}_{p}$ over the Galois extension $K_{\infty} / K$ discussed above. We take $M$ to be the module $\mathcal{S}_{A}^{\vee} / \mathcal{S}_{A}^{\vee}(p)$. It is conjectured that the module $M=\mathcal{S}_{A}^{\vee} / \mathcal{S}_{A}^{\vee}(p)$ is in the category $\mathfrak{n}_{H}(G)$ (see [Coates et al. 2005, Conjecture 5.1]) and there are examples where this conjecture is satisfied (see [loc. cit.]).

(2) Let us choose a $p$-th power free integer $m \geq 2$. Put $K=\mathbb{Q}\left(\mu_{p}\right), K_{\text {cyc }}=\mathbb{Q}\left(\mu_{p^{\infty}}\right)$ and $K_{\infty}=\bigcup_{n=1}^{\infty} K_{\text {cyc }}\left(m^{1 / p^{n}}\right)$. Such an extension $K_{\infty} / K$ is called a false-Tate curve extension. We denote $\operatorname{Gal}\left(K_{\infty} / K\right), \operatorname{Gal}\left(K_{\infty} / K_{\text {cyc }}\right)$ and $\operatorname{Gal}\left(K_{\text {cyc }} / K\right)$ by $G, H$ and $\Gamma$ respectively. Note that we have $G \cong \mathbb{Z}_{p} \rtimes \mathbb{Z}_{p}, H \cong \mathbb{Z}_{p}$ and $\Gamma \cong \mathbb{Z}_{p}$. Again the pair $H \subset G$ satisfies the condition $(\mathrm{G})$.

Let us consider the Pontryagin dual $\mathcal{S}_{A}^{\vee}$ of the Selmer group $\mathcal{S}_{A}$ of the Galois representation $A=T \otimes \mathbb{Q}_{p} / \mathbb{Z}_{p}$ over a Galois extension $K_{\infty} / K$ discussed above. We take $M$ to be the module $\mathcal{S}_{A}^{\vee} / \mathcal{S}_{A}^{\vee}(p)$. Under certain assumptions on $A$, it is expected that $\mathcal{S}_{A}^{\vee} / \mathcal{S}_{A}^{\vee}(p)$ will be in $\mathfrak{n}_{H}(G)$. We refer to [Hachimori and Venjakob 2003] for some examples of $\mathcal{S}_{A}^{\vee} / \mathcal{S}_{A}^{\vee}(p)$ which are in $\mathfrak{n}_{H}(G)$.

(3) Let $K$ be an imaginary quadratic field in which a rational prime $p \neq 2$ splits. Let $K_{\infty}$ be the unique $\mathbb{Z}_{p}^{\oplus 2}$-extension of $K$. Let $G=\operatorname{Gal}\left(K_{\infty} / K\right)$ and $H=$ $\operatorname{Gal}\left(K_{\infty} / K_{\text {cyc }}\right)$. Once again the pair $H \subset G$ satisfies the condition $(\mathrm{G})$.

For the Pontryagin dual $\mathcal{S}_{A}^{\vee}$ of the Selmer group $\mathcal{S}_{A}$ of the Galois representation $A=T \otimes \mathbb{Q}_{p} / \mathbb{Z}_{p}$ over a Galois extension $K_{\infty} / \mathbb{Q}$ in this commutative two-variable situation, similar phenomena as above are expected and we take $M$ to be the module $\mathcal{S}_{A}^{\vee} / \mathcal{S}_{A}^{\vee}(p)$.

In a forthcoming joint work of two of us [Jha and Ochiai $\geq 2016$ ], the Main Theorem above will be applied to establish the functional equation of Selmer groups for general $p$-adic representations over a general noncommutative $p$-adic Lie extension. This is a partial motivation for our present work for two of us. Note that the third author proved the functional equation of Selmer groups for elliptic curves over 
false-Tate curve extension (see [Zábrádi 2008]) and for non-CM elliptic curves in $\mathrm{GL}_{2}$-extension (see [Zábrádi 2010]). But the main method of the papers [Zábrádi $2008 ; 2010$ ] is not based on the twisting lemma.

Notation. Unless otherwise specified, all modules over $\Lambda_{\mathcal{O}}(G)$ are considered as left modules. Throughout the paper we fix a topological generator $\gamma$ of $\Gamma$.

\section{Preliminary Theorem}

In this section, we formulate and prove the Preliminary Theorem below, which gives the same conclusion as the Main Theorem under stronger assumptions (i.e., the hypothesis $(\mathrm{H})$ and nonexistence of nontrivial element of order $p$ in $G$ ). In the next section, our Main Theorem is deduced from the Preliminary Theorem and the Key Lemma which is given in the next section.

Preliminary Theorem. Let $G$ be a compact p-adic Lie group without any element of order $p$ and let $H$ be a closed normal subgroup such that $G / H$ is isomorphic to $\Gamma$. Let $M$ be a finitely generated torsion $\Lambda_{\mathcal{O}}(G)$-module satisfying the following condition.

(H) There is a $\Lambda_{\mathcal{O}}(H)$-linear homomorphism $M \rightarrow \mathbb{Z}_{p} \llbracket H \rrbracket^{\oplus d}$ that induces an isomorphism $M \otimes_{\mathbb{Z}_{p}} \mathbb{Q}_{p} \stackrel{\sim}{\rightarrow}\left(\mathbb{Z}_{p} \llbracket H \rrbracket \otimes_{\mathbb{Z}_{p}} \mathbb{Q}_{p}\right)^{\oplus d}$ after taking $\otimes_{\mathbb{Z}_{p}} \mathbb{Q}_{p}$.

Then there exists a continuous character $\rho: \Gamma \rightarrow \mathbb{Z}_{p}^{\times}$such that the largest $U$ coinvariant quotient $M(\rho)_{U}$ of $M(\rho)$ is finite for every open normal subgroup $U$ of $G$.

Before going into the proof of the Preliminary Theorem, we collect some basic results in noncommutative Iwasawa theory which are relevant for the article.

Lemma 1. Let $H \subset G$ be a pair satisfying the condition $(G)$ and let $M$ be a finitely generated $\Lambda_{\mathcal{O}}(G)$-module which satisfies the condition $(H)$. Then there exists a matrix $A \in M_{d}\left(\mathbb{Z}_{p} \llbracket H \rrbracket \otimes_{\mathbb{Z}_{p}} \mathbb{Q}_{p}\right)$ such that $M \otimes_{\mathbb{Z}_{p}} \mathbb{Q}_{p}$ is isomorphic to

$$
\left(\mathbb{Z}_{p} \llbracket G \rrbracket \otimes_{\mathbb{Z}_{p}} \mathbb{Q}_{p}\right)^{\oplus d} /\left(\mathbb{Z}_{p} \llbracket G \rrbracket \otimes_{\mathbb{Z}_{p}} \mathbb{Q}_{p}\right)^{\oplus d}\left(\tilde{\gamma} \mathbf{1}_{d}-A\right),
$$

where $\gamma$ is a topological generator of $\Gamma$ and $\tilde{\gamma} \in G$ is a fixed lift of $\gamma$ and elements in $\left(\mathbb{Z}_{p} \llbracket G \rrbracket \otimes_{\mathbb{Z}_{p}} \mathbb{Q}_{p}\right)^{\oplus d}$ are regarded as row vectors.

Proof. Let us take a basis $\boldsymbol{v}_{1}, \ldots, \boldsymbol{v}_{d}$ of the free $\mathbb{Z}_{p} \llbracket H \rrbracket \otimes_{\mathbb{Z}_{p}} \mathbb{Q}_{p}$-module $M \otimes_{\mathbb{Z}_{p}} \mathbb{Q}_{p}$. Through the isomorphism $M \otimes_{\mathbb{Z}_{p}} \mathbb{Q}_{p} \stackrel{\sim}{\rightarrow}\left(\mathbb{Z}_{p} \llbracket H \rrbracket \otimes_{\mathbb{Z}_{p}} \mathbb{Q}_{p}\right)^{\oplus d}$ fixed by the condition (H), $\tilde{\gamma}$ acts on $M$. Thus we define a matrix $A=\left(a_{i j}\right)_{1 \leq i, j \leq d} \in M_{d}\left(\mathbb{Z}_{p} \llbracket H \rrbracket \otimes_{\mathbb{Z}_{p}} \mathbb{Q}_{p}\right)$ by

$$
\tilde{\gamma} \cdot \boldsymbol{v}_{i}=\sum_{1 \leq j \leq d} a_{j i} \boldsymbol{v}_{j}
$$


We denote the module presented in (1) by $N_{A}$. By construction, we have a $\mathbb{Z}_{p} \llbracket H \rrbracket \otimes_{\mathbb{Z}_{p}} \mathbb{Q}_{p}$-linear isomorphism $\left(\mathbb{Z}_{p} \llbracket H \rrbracket \otimes_{\mathbb{Z}_{p}} \mathbb{Q}_{p}\right)^{\oplus d} \stackrel{\sim}{\rightarrow} N_{A}$ on which $\tilde{\gamma}$ acts in the same manner as the action of $\tilde{\gamma}$ on $M \otimes_{\mathbb{Z}_{p}} \mathbb{Q}_{p}$.

We denote by $\mathcal{U}$ the set of all open normal subgroups $U$ of $G$. We remark that the set $\mathcal{U}$ is a countable set since $G$ is profinite and has a countable base at the identity.

Lemma 2. For any $U \in \mathcal{U}, \mathbb{Z}_{p}[G / U] \otimes_{\mathbb{Z}_{p}} \overline{\mathbb{Q}}_{p}$ is isomorphic to a finite number of products of matrix algebras $\prod_{i=1}^{k(U)} M_{r_{i}}\left(\overline{\mathbb{Q}}_{p}\right)$.

Proof. First of all, the algebra $\mathbb{Z}_{p}[G / U] \otimes_{\mathbb{Z}_{p}} \mathbb{Q}_{p} \cong \mathbb{Q}_{p}[G / U]$ is a semisimple algebra over $\mathbb{Q}_{p}$ since $G / U$ is a finite group and $\mathbb{Q}_{p}$ is of characteristic 0 . We have an isomorphism

$$
\mathbb{Q}_{p}[G / U] \cong \prod_{i=1}^{l_{n}} M_{s_{i}}\left(D_{i}\right),
$$

where $D_{i}$ is a finite dimensional division algebra over $\mathbb{Q}_{p}$. For each $i$, the center $K_{i}$ of $D_{i}$ is a finite extension of $\mathbb{Q}_{p}$. It is well-known that $\operatorname{dim}_{K_{i}} D_{i}$ is a square of some natural number $t_{i}$ and $D_{i} \otimes_{K_{i}} \overline{\mathbb{Q}}_{p}$ is isomorphic to $M_{t_{i}}\left(\overline{\mathbb{Q}}_{p}\right)$. Thus $M_{s_{i}}\left(D_{i}\right) \otimes \overline{\mathbb{Q}}_{p}$ is isomorphic to $\left[K_{i}: \mathbb{Q}_{p}\right]$ copies of $M_{s_{i}+t_{i}}\left(\overline{\mathbb{Q}}_{p}\right)$. The lemma follows immediately from this.

Proof of the Preliminary Theorem. First, we remark that for an open normal subgroup $U$ of $G$, we have

$$
M(\rho)_{U} \text { is finite if and only if } M(\rho)_{U} \otimes_{\mathbb{Z}_{p}} \mathbb{Q}_{p}=0 .
$$

Since the operation of taking the base extension $\otimes_{\mathbb{Z}_{p}} \mathbb{Q}_{p}$ commutes with the operation of taking the largest $U$-coinvariant quotient, by Lemma 1 we have

$$
\begin{aligned}
M(\rho)_{U} \otimes_{\mathbb{Z}_{p}} \mathbb{Q}_{p} & \\
& \cong\left(\mathbb{Z}_{p}[G / U] \otimes_{\mathbb{Z}_{p}} \mathbb{Q}_{p}\right)^{\oplus d} /\left(\mathbb{Z}_{p}[G / U] \otimes_{\mathbb{Z}_{p}} \mathbb{Q}_{p}\right)^{\oplus d}\left(\tilde{\gamma}_{U}^{\oplus d}-A_{U}(\rho)\right),
\end{aligned}
$$

where we denote the projection of $\tilde{\gamma} \in G$ to $G / U$ by $\tilde{\gamma}_{U}$. Here, the matrix $A_{U}(\rho) \in$ $M_{d}\left(\mathbb{Z}_{p}[G / U] \otimes_{\mathbb{Z}_{p}} \mathbb{Q}_{p}\right)$ is defined as the image of $\rho(\gamma)^{-1} A \in M_{d}\left(\mathbb{Z}_{p} \llbracket H \rrbracket \otimes_{\mathbb{Z}_{p}} \mathbb{Q}_{p}\right)$ via the composite map

$$
M_{d}\left(\mathbb{Z}_{p} \llbracket H \rrbracket \otimes_{\mathbb{Z}_{p}} \mathbb{Q}_{p}\right) \longrightarrow M_{d}\left(\mathbb{Z}_{p} \llbracket G \rrbracket \otimes_{\mathbb{Z}_{p}} \mathbb{Q}_{p}\right) \longrightarrow M_{d}\left(\mathbb{Z}_{p}[G / U] \otimes_{\mathbb{Z}_{p}} \mathbb{Q}_{p}\right) .
$$

Taking the base extension $\otimes_{\mathbb{Q}_{p}} \overline{\mathbb{Q}}_{p}$ of the isomorphism (3), we have a $\overline{\mathbb{Q}}_{p}$-linear isomorphism by Lemma 2:

$$
M(\rho)_{U} \otimes_{\mathbb{Z}_{p}} \overline{\mathbb{Q}}_{p} \cong \prod_{i=1}^{k(U)} M_{r_{i}}\left(\overline{\mathbb{Q}}_{p}\right)^{\oplus d} / M_{r_{i}}\left(\overline{\mathbb{Q}}_{p}\right)^{\oplus d}\left(\gamma_{U, i}^{\oplus d}-A_{U, i}(\rho)\right)
$$


where $\gamma_{U, i} \in \operatorname{Aut}_{\overline{\mathbb{Q}}_{p}}\left(M_{r_{i}}\left(\overline{\mathbb{Q}}_{p}\right)\right)$ and $A_{U, i}(\rho) \in \operatorname{End}_{\overline{\mathbb{Q}}_{p}}\left(M_{r_{i}}\left(\overline{\mathbb{Q}}_{p}\right)^{\oplus d}\right)$ are defined as follows. We consider the base extension to $\overline{\mathbb{Q}}_{p}$ of

$$
\tilde{\gamma}_{U} \in \operatorname{Aut}_{\mathbb{Z}_{p}[G / U] \otimes_{\mathbb{Z}_{p}} \mathbb{Q}_{p}}\left(\mathbb{Z}_{p}[G / U] \otimes_{\mathbb{Z}_{p}} \mathbb{Q}_{p}\right) \subset \operatorname{Aut}_{\mathbb{Q}_{p}}\left(\mathbb{Z}_{p}[G / U] \otimes_{\mathbb{Z}_{p}} \mathbb{Q}_{p}\right) .
$$

This is an element of $\operatorname{Aut}_{\overline{\mathbb{Q}}_{p}}\left(\prod_{i=1}^{k(U)} M_{r_{i}}\left(\overline{\mathbb{Q}}_{p}\right)\right)$. We denote the projection of this element to the $i$-th component by $\gamma_{U, i}$. The base extension to $\overline{\mathbb{Q}}_{p}$ of

$$
A_{U}(\rho) \in M_{d}\left(\mathbb{Z}_{p}[G / U] \otimes_{\mathbb{Z}_{p}} \mathbb{Q}_{p}\right) \subset \operatorname{End}_{\mathbb{Q}_{p}}\left(\left(\mathbb{Z}_{p}[G / U] \otimes_{\mathbb{Z}_{p}} \mathbb{Q}_{p}\right)^{\oplus d}\right)
$$

is an element of $\operatorname{End}_{\overline{\mathbb{Q}}_{p}}\left(\prod_{i=1}^{k(U)} M_{r_{i}}\left(\overline{\mathbb{Q}}_{p}\right)^{\oplus d}\right)$, and we denote the projection of this element to the $i$-th component by $A_{U, i}(\rho)$.

Now, we denote by $A_{U, i}$ the element $A_{U, i}(\mathbf{1})$. We remark that $A_{U, i}(\rho)$ is equal to $\rho(\gamma)^{-1} A_{U, i}$ for any continuous character $\rho: \Gamma \rightarrow \mathbb{Z}_{p}^{\times}$. We define $\mathrm{EV}_{U, i}$ to be the set of roots of the characteristic polynomial

$$
P_{U, i}(T):=\operatorname{det}\left(\gamma_{U, i}^{\oplus d}-A_{U, i} T\right) .
$$

Since $\gamma_{U, i}^{\oplus d}$ is an automorphism, the polynomial $P_{U, i}(T)$ is not zero. Hence $\mathrm{EV}_{U, i}$ is a finite set. We denote the union of $\mathrm{EV}_{U, i}$ for $1 \leq i \leq k(U)$ by $\mathrm{EV}_{U}$, which is again a finite set. If $\rho(\gamma)^{-1}$ is not contained in $\mathrm{EV}_{U} \cap \mathbb{Z}_{p}^{\times}$, the module in (4) is zero and hence the module in (3) is zero. Now, we denote by $\mathrm{EV}_{M}$ the union of $\mathrm{EV}_{U} \cap \mathbb{Z}_{p}^{\times}$over all $U \in \mathcal{U}$. Since $\mathcal{U}$ is a countable set, $\mathrm{EV}_{M}$ is a countable set. Thus $\mathbb{Z}_{p}^{\times} \backslash \mathrm{EV}_{M}$ is nonempty since $\mathbb{Z}_{p}^{\times}$is uncountable. By choosing $\rho(\gamma)^{-1} \in \mathbb{Z}_{p}^{\times} \backslash \mathrm{EV}_{M}$, we complete the proof.

\section{Proof of the Main Theorem}

In this section, we prove the Main Theorem, which relies on the following result.

Key Lemma. Let $G$ be a compact p-adic Lie group without any element of order $p$ and let $H$ be a closed subgroup such that $G / H$ is isomorphic to $\Gamma$. Let $M$ be $\Lambda_{\mathcal{O}}(G)$-module which is finitely generated over $\Lambda_{\mathcal{O}}(H)$. Then, there exists an open subgroup $G_{0} \subset G$ containing $H, a \Lambda_{\mathcal{O}}\left(G_{0}\right)$-module $N$ which is a free $\Lambda_{\mathcal{O}}(H)$-module of finite rank, and a surjective $\Lambda_{\mathcal{O}}\left(G_{0}\right)$-linear homomorphism $N \rightarrow M$.

Proof. We denote by $I$ the Jacobson radical of $\Lambda_{\mathcal{O}}(H)$. Note that I is a two sided ideal of $\Lambda_{\mathcal{O}}(H)$ such that we have $\Lambda_{\mathcal{O}}(H) / I \cong \mathbb{F}_{q}$, where $\mathbb{F}_{q}$ is the residue field of $\mathcal{O}$. We also have $\Lambda_{\mathcal{O}}(G) / I \cong \mathbb{F}_{q} \llbracket \Gamma \rrbracket$ by definition.

Let us take a system of generators $m_{1}, \ldots, m_{d}$ of $M$ as a $\Lambda_{\mathcal{O}}(H)$-module. Note that $M$ is equipped with a topology obtained by a natural $\Lambda_{\mathcal{O}}(H)$-module structure. The set $\left\{I^{n} M\right\}_{n \in \mathbb{N}}$ forms a system of open neighborhoods of $M$. 
Choose a topological generator $\gamma$ of $\Gamma$ and take a lift $\tilde{\gamma} \in G$ of $\gamma$. By continuity of the action of $G$ on $M$, the following two conditions hold true simultaneously for a sufficiently large integer $n$ :

(i) We have $\left(\tilde{\gamma}^{p^{n}}-1\right) m_{i} \in I M$ for any $i$ with $1 \leq i \leq d$.

(ii) The conjugate action of $\tilde{\gamma}^{p}$ on $I / I^{2}$ is trivial.

We will choose and fix a natural number $n$ satisfying the conditions (i) and (ii). Then we define $G_{0}$ to be the preimage of $\Gamma^{p^{n}}$ by the surjection $G \rightarrow \Gamma$. By definition, $G_{0}$ is an open subgroup of $G$ which contain $H$.

Let us consider the set $\left\{a_{i j} \in I\right\}_{1 \leq i, j \leq d}$ such that we have $\left(\tilde{\gamma}^{p^{n}}-1\right) m_{j}=$ $\sum_{i=1}^{d} a_{i j} m_{i}$. We consider $F$ (resp. $\left.F^{\prime}\right)$ which is a free $\Lambda_{\mathcal{O}}\left(G_{0}\right)$-module of rank $d$ equipped with a system of generators $f_{1}, \ldots, f_{d}$ (resp. $f_{1}^{\prime}, \ldots, f_{d}^{\prime}$ ). We consider a $\Lambda_{\mathcal{O}}\left(G_{0}\right)$-linear homomorphism

$$
\varphi: F^{\prime} \longrightarrow F, \quad f_{j}^{\prime} \mapsto\left(\tilde{\gamma}^{p^{n}}-1\right) f_{j}-\sum_{i=1}^{d} a_{i j} f_{i} .
$$

We define a $\Lambda_{\mathcal{O}}\left(G_{0}\right)$-module $N$ to be the cokernel of the map $\varphi$ above.

Claim. For each $i$ with $1 \leq i \leq d$, we denote the image of $f_{i}$ by $\bar{f}_{i}$. Then the $\Lambda_{\mathcal{O}}\left(G_{0}\right)$-module $N$ is a free $\Lambda_{\mathcal{O}}(H)$-module of finite rank $d$ with a system of generators $\bar{f}_{1}, \ldots, \bar{f}_{d}$.

If the claim holds true, a $\Lambda_{\mathcal{O}}\left(G_{0}\right)$-linear homomorphism $N \rightarrow M$ sending $\bar{f}_{i}$ to $m_{i}$ for each $i$ is surjective. Since $N$ is free over $\Lambda_{\mathcal{O}}(H)$, this is what we want. Thus it remains only to prove the claim.

By applying the functor $\Lambda_{\mathcal{O}}\left(G_{0}\right) / I \Lambda_{\mathcal{O}}\left(G_{0}\right) \otimes_{\Lambda_{\mathcal{O}}\left(G_{0}\right)} \cdot$ to the map $\varphi$, and noting that

$$
\Lambda_{\mathcal{O}}\left(G_{0}\right) / I \cong \mathbb{F}_{q} \llbracket \Gamma \rrbracket,
$$

we obtain

$$
\varphi_{I}: \oplus_{j=1}^{d} \mathbb{F}_{q} \llbracket \Gamma^{p^{n}} \rrbracket f_{j}^{\prime} \stackrel{\times\left(\tilde{\gamma}^{p^{n}}-1\right)}{\longrightarrow} \oplus_{j=1}^{d} \mathbb{F}_{q} \llbracket \Gamma^{p^{n}} \rrbracket f_{j} .
$$

Since $N / I N$ is isomorphic to the cokernel of the above map $\varphi_{I}, N / I N$ is a free $\mathbb{F}_{q}$ module of rank $d$. By applying the topological Nakayama lemma (see [Balister and Howson 1997, Corollary in §3]) to the compact $\Lambda_{\mathcal{O}}(H)$-module $N, N$ is generated by $\bar{f}_{1}, \ldots, \bar{f}_{d}$ over $\Lambda_{\mathcal{O}}(H)$. We will prove that $N$ is free of rank $d$ over $\Lambda_{\mathcal{O}}(H)$ with this system of generators. Let $r$ be an arbitrary natural number. Since we have a natural surjection from the $r$-fold tensor product of $I / I^{2}$ to $I^{r} / I^{r+1}$, the conjugate action of $\tilde{\gamma}^{p^{n}}$ on $I^{r} / I^{r+1}$ is also trivial. Thus, by applying the functor $I^{r} / I^{r+1} \Lambda_{\mathcal{O}}\left(G_{0}\right) \otimes_{\Lambda_{\mathcal{O}}\left(G_{0}\right)} \cdot$ to the map $\varphi$, we obtain a $\Lambda_{\mathcal{O}}\left(G_{0}\right)$-linear map

$$
\varphi \otimes I^{r} / I^{r+1}: I^{r} F^{\prime} / I^{r+1} F^{\prime} \longrightarrow I^{r} F / I^{r+1} F
$$


which is again defined as a multiplication of $\left(\tilde{\gamma}^{p^{n}}-1\right)$. This proves

$$
\operatorname{dim}_{\mathbb{F}_{q}} N / I^{s} N=\sum_{r=0}^{s-1} \operatorname{dim}_{\mathbb{F}_{q}} I^{r} N / I^{r+1} N=\sum_{r=0}^{s-1} \operatorname{dim}_{\mathbb{F}_{q}}\left(I^{r} / I^{r+1}\right)^{\oplus d} .
$$

Thus the cardinality of $N / I^{s} N$ is equal to the cardinality of $\left(\Lambda_{\mathcal{O}}(H) / I\right)^{\oplus d}$ for any natural number $s$, which implies that $N$ is free of rank $d$ over $\Lambda_{\mathcal{O}}(H)$. This completes the proof of the claim.

Proof of Main Theorem. We will use the Key Lemma and the Preliminary Theorem to prove the Main Theorem in two steps.

First, we consider the situation where $G$ is a compact $p$-adic Lie group without any element of order $p$ and $H$ is a closed subgroup such that $G / H$ is isomorphic to $\Gamma$. Thus we dropped the assumption $(\mathrm{H})$ of the Preliminary Theorem but we still keep the assumption of nonexistence of a nontrivial element of order $p$ in $G$.

Let $M$ be $\Lambda_{\mathcal{O}}(G)$-module which is finitely generated over $\Lambda_{\mathcal{O}}(H)$. By the Key Lemma, for a sufficiently large natural number $n$, we have a surjective $\Lambda_{\mathcal{O}}\left(G_{0}\right)$ linear homomorphism $N \rightarrow M$ from a free $\Lambda_{\mathcal{O}}(H)$-module $N$ of finite rank. Here $G_{0}$ is a unique open subgroup $G_{0} \subset G$ of index $p^{n}$ containing $H$. Note that the module $N$ satisfies condition $(\mathrm{H})$ of the Preliminary Theorem. We thus find a continuous character $\rho_{0}: \Gamma^{p^{n}} \rightarrow \mathbb{Z}_{p}^{\times}$such that $N\left(\rho_{0}\right)_{U_{0}}$ is finite for any open normal subgroup $U_{0}$ of $G_{0}$. By the proof of the Preliminary Theorem, we can choose uncountably many such $\rho_{0}$. Thus, we see that we can take $\rho_{0}$ as above so that the value of $\rho_{0}$ is contained in a open subgroup $1+p^{n} \mathbb{Z}_{p}$ of $\mathbb{Z}_{p}^{\times}$. Then, we take a continuous character $\rho: \Gamma \rightarrow \mathbb{Z}_{p}^{\times}$whose restriction to $\Gamma^{p^{n}}$ coincides with $\rho_{0}$. The twist $M(\rho)$ with this character is what we want in our Main Theorem. In fact, for any open normal subgroup $U$ of $G$, we have a surjection $N\left(\rho_{0}\right)_{U_{0}} \rightarrow M(\rho)_{U}$ taking an open normal subgroup $U_{0}$ of $G_{0}$ contained in $U$. Since $N\left(\rho_{0}\right)_{U_{0}}$ is finite by the Preliminary Theorem, $M(\rho)_{U}$ must be finite. Thus we finished the proof of our Main Theorem under the assumption of nonexistence of a nontrivial element of order $p$ in $G$.

Now we deduce our Main Theorem assuming that it is true under the assumption of nonexistence of a nontrivial element of order $p$ in $G$. We consider the situation where $G$ is a compact $p$-adic Lie group with elements of order $p$ and $H$ is a closed subgroup such that $G / H$ is isomorphic to $\Gamma$. Let $M$ be a $\Lambda_{\mathcal{O}}(G)$-module which is finitely generated over $\Lambda_{\mathcal{O}}(H)$. Let $G^{\prime}$ be a uniform open normal subgroup of $G$ (see [Lazard 1965, Chapter III, $\S(3.1)]$ ), which is automatically without any elements of order $p$. Let $H^{\prime}$ be the intersection of $H$ and $G^{\prime}$. Since $M$ is finitely generated over $\Lambda_{\mathcal{O}}(H)$ and since $H^{\prime}$ is of finite index in $H, M$ is finitely generated over $\Lambda_{\mathcal{O}}\left(H^{\prime}\right)$. According to the result in our first step, there exist a continuous character $\rho^{\prime}: G^{\prime} / H^{\prime} \rightarrow \mathbb{Z}_{p}^{\times}$such that $M\left(\rho^{\prime}\right)_{U^{\prime}}$ is finite for every open subgroup $U^{\prime}$ 
of $G^{\prime}$. Note that $G^{\prime} / H^{\prime}$ is naturally regarded as an open subgroup of $G / H$. Thus by choosing $\rho^{\prime}$ so that the image of $\rho^{\prime}$ is small enough in $\mathbb{Z}_{p}^{\times}$compared to the index of $G^{\prime} / H^{\prime}$ in $G / H$, there exists a continuous character $\rho: G / H \rightarrow \mathbb{Z}_{p}^{\times}$whose restriction to $G^{\prime} / H^{\prime}$ coincides with $\rho^{\prime}$. Now for any open normal subgroup $U$ of $G$, we take an open normal subgroup $U^{\prime}$ of $G^{\prime}$ which is contained in $U$. We have a natural map $M\left(\rho^{\prime}\right)_{U^{\prime}} \rightarrow M(\rho)_{U}$, where $M\left(\rho^{\prime}\right)_{U^{\prime}}$ is finite by the choice of $\rho^{\prime}$ and by our discussion above. Unlike in the first step, $M\left(\rho^{\prime}\right)_{U^{\prime}} \rightarrow M(\rho)_{U}$ is not necessarily surjective. However, the cokernel of this map is still finite by construction. We thus deduce that $M(\rho)_{U}$ is finite, which completes the proof of the Main Theorem.

Remark ( $p$-torsion modules). For a compact $p$-adic Lie group $G$ without any element of order $p$, it is well-known that $\Lambda_{O}(G)$ is left and right noetherian. Let $N$ be a finitely generated $p$-primary torsion left $\Lambda_{\mathcal{O}}(G)$ module. Then, we have $N=N\left[p^{r}\right]$ for some $r \in \mathbb{N}$. For any open normal subgroup $U$ of $G, N_{U}$ is a finitely generated $\mathbb{Z} / p^{r} \mathbb{Z}[G / U]$ module. In other words, $N_{U}$ is always finite when $N$ is of $p$-primary torsion.

For a finitely generated torsion $\Lambda_{\mathcal{O}}(G)$ module $M$, we consider the exact sequence

$$
0 \longrightarrow M(p) \longrightarrow M \longrightarrow M / M(p) \longrightarrow 0,
$$

where $M(p)$ is the largest $p$-primary torsion submodule of $M$. Then, from the preceding discussion, it is clear that in the situation of the Main Theorem, for any continuous $\rho: \Gamma \rightarrow \mathbb{Z}_{p}^{\times}$and for any open normal subgroup $U$ of $G, M(\rho)_{U}$ is finite if and only if $((M / M(p))(\rho))_{U}$ is finite.

In particular, when we want to apply the Main Theorem to arithmetic situations coming from Selmer groups of certain Galois modules $A$, we remark that $\mathcal{S}_{A}^{\vee}(\rho)_{U}$ is finite if and only if $\left(\left(\mathcal{S}_{A}^{\vee} / \mathcal{S}_{A}^{\vee}(p)\right)(\rho)\right)_{U}$ is finite.

\section{Acknowledgements}

A part of the paper was done when S. Jha visited Osaka University. He thanks Osaka University for their hospitality. The paper was finalized when T. Ochiai stayed at the Indian Institute of Technology Kanpur (IITK) on September 2015. He thanks IITK for their hospitality. We are grateful to Prof. John Coates for encouragement and invaluable suggestions leading to improvements on an earlier draft of the paper.

\section{References}

[Balister and Howson 1997] P. N. Balister and S. Howson, "Note on Nakayama's lemma for compact $\Lambda$-modules”, Asian J. Math. 1:2 (1997), 224-229. MR 1491983 Zbl 0904.16019

[Coates et al. 2005] J. Coates, T. Fukaya, K. Kato, R. Sujatha, and O. Venjakob, "The GL2 main conjecture for elliptic curves without complex multiplication", Inst. Hautes Études Sci. Publ. Math. 101 (2005), 163-208. MR 2217048 Zbl 1108.11081 
[Greenberg 1989] R. Greenberg, "Iwasawa theory for $p$-adic representations", pp. 97-137 in Algebraic number theory, edited by J. Coates et al., Adv. Stud. Pure Math. 17, Academic Press, Boston, 1989. MR 1097613 Zbl 0739.11045

[Hachimori and Venjakob 2003] Y. Hachimori and O. Venjakob, "Completely faithful Selmer groups over Kummer extensions”, Doc. Math. Extra Vol. (2003), 443-478. MR 2046605 Zbl 1117.14046

[Jha and Ochiai $\geq 2016$ ] S. Jha and T. Ochiai, "Functional equation of Selmer groups over $p$-adic Lie extension", in preparation.

[Lazard 1965] M. Lazard, "Groupes analytiques p-adiques”, Inst. Hautes Études Sci. Publ. Math. 26 (1965), 5-219. MR 0209286 Zbl 0139.02302

[Perrin-Riou 2003] B. Perrin-Riou, "Groupes de Selmer et accouplements: cas particulier des courbes elliptiques”, Doc. Math. Extra Vol. (2003), 725-760. MR 2046613 Zbl 1142.11337

[Zábrádi 2008] G. Zábrádi, "Characteristic elements, pairings and functional equations over the false Tate curve extension”, Math. Proc. Cambridge Philos. Soc. 144:3 (2008), 535-574. MR 2418704 Zbl 1243.11106

[Zábrádi 2010] G. Zábrádi, "Pairings and functional equations over the $\mathrm{GL}_{2}$-extension", Proc. Lond. Math. Soc. (3) 101:3 (2010), 893-930. MR 2734964 Zbl 1272.11119

Communicated by John Henry Coates

Received 2015-10-21 Revised 2015-12-22 Accepted 2016-02-01

jhasom@iitk.ac.in

Department of Mathematics and Statistics, Indian Institute of Technology Kanpur, Kanpur 208016, India

ochiai@math.sci.osaka-u.ac.jp Department of Mathematics, Graduate School of Science, Osaka University, Machikaneyama 1-1, Toyonaka, Osaka 5600043, Japan

zger@cs.elte.hu

Department of Algebra and Number Theory, Mathematical Institute, Eötvös Loránd University, Bertalan Lajos utca 11, 1111 Budapest, Hungary 


\section{Algebra \& Number Theory}

msp.org/ant

\section{EDITORS}

MANAGING EDITOR

Bjorn Poonen

Massachusetts Institute of Technology

Cambridge, USA

\author{
EDITORIAL BOARD CHAIR \\ David Eisenbud \\ University of California \\ Berkeley, USA
}

\section{BOARD OF EDITORS}

Georgia Benkart

Dave Benson

Richard E. Borcherds

John H. Coates

J-L. Colliot-Thélène

Brian D. Conrad

Hélène Esnault

Hubert Flenner

Sergey Fomin

Edward Frenkel

Andrew Granville

Joseph Gubeladze

Roger Heath-Brown

Craig Huneke

Kiran S. Kedlaya

János Kollár

Yuri Manin

Philippe Michel
University of Wisconsin, Madison, USA

University of Aberdeen, Scotland

University of California, Berkeley, USA

University of Cambridge, UK

CNRS, Université Paris-Sud, France

Stanford University, USA

Freie Universität Berlin, Germany

Ruhr-Universität, Germany

University of Michigan, USA

University of California, Berkeley, USA

Université de Montréal, Canada

San Francisco State University, USA

Oxford University, UK

University of Virginia, USA

Univ. of California, San Diego, USA

Princeton University, USA

Northwestern University, USA

École Polytechnique Fédérale de Lausanne
Susan Montgomery

Shigefumi Mori

Raman Parimala

Jonathan Pila

Anand Pillay

Victor Reiner

Peter Sarnak

Joseph H. Silverman

Michael Singer

Vasudevan Srinivas

J. Toby Stafford

Ravi Vakil

Michel van den Bergh

Marie-France Vignéras

Kei-Ichi Watanabe

Efim Zelmanov

Shou-Wu Zhang
University of Southern California, USA

RIMS, Kyoto University, Japan

Emory University, USA

University of Oxford, UK

University of Notre Dame, USA

University of Minnesota, USA

Princeton University, USA

Brown University, USA

North Carolina State University, USA

Tata Inst. of Fund. Research, India

University of Michigan, USA

Stanford University, USA

Hasselt University, Belgium

Université Paris VII, France

Nihon University, Japan

University of California, San Diego, USA

Princeton University, USA

\section{PRODUCTION}

production@msp.org

Silvio Levy, Scientific Editor

See inside back cover or msp.org/ant for submission instructions.

The subscription price for 2016 is US \$/year for the electronic version, and \$/year (+\$, if shipping outside the US) for print and electronic. Subscriptions, requests for back issues and changes of subscribers address should be sent to MSP.

Algebra \& Number Theory (ISSN 1944-7833 electronic, 1937-0652 printed) at Mathematical Sciences Publishers, 798 Evans Hall \#3840, c/o University of California, Berkeley, CA 94720-3840 is published continuously online. Periodical rate postage paid at Berkeley, CA 94704, and additional mailing offices.

ANT peer review and production are managed by EditFLOW ${ }^{\circledR}$ from MSP.

\section{PUBLISHED BY}

mathematical sciences publishers

nonprofit scientific publishing

http://msp.org/

(C) 2016 Mathematical Sciences Publishers 


\section{Algebra \& Number Theory}

Volume $10 \quad$ No. $3 \quad 2016$

Group schemes and local densities of ramified hermitian lattices in residue characteristic 2: Part I

SUNGMUN CHO

Presentation of affine Kac-Moody groups over rings

DANIEL ALLCOCK

Discriminant formulas and applications

Kenneth Chan, Alexander A. Young and James J. Zhang

LUIS E. GARCIA

Multiple period integrals and cohomology

RoElof W. BRUGGEMAN and YoungJu CHOIE

The existential theory of equicharacteristic henselian valued fields

SYLVY ANSCOMBE and ARNO FEHM

SOMNATH JHA, TADASHI OCHIAI and GERGELY ZÁBRÁDI 